\title{
Uma Proposta para Uso de Computação Desplugada no Ensino Híbrido
}

\author{
Quesia Araújo $^{1}$, Anderson Corrêa de Lima ${ }^{1}$, Amaury Antônio de Castro Junior ${ }^{1}$, \\ Wilk Oliveira $^{2}$ \\ ${ }^{1}$ Universidade Federal de Mato Grosso do Sul (UFMS) \\ Av. Costa e Silva, s/nº, Bairro Universitário - Campo Grande, MS - Brasil \\ ${ }^{2}$ Instituto de Ciências Matemáticas e de Computação (ICMC) \\ Universidade de São Paulo (USP) - São Carlos, SP - Brasil \\ \{quesia.araujo, anderson.lima, amaury.junior\}@ufms.br, wilk.oliveira@usp.br
}

\begin{abstract}
Unplugged Computing has been a reference for teaching Computing Education subjects. However, the advent of the Covid-19 pandemic changed this scenario. Thus, this paper aims to present a set of criteria that can guide the choice and adaptation of unplugged activities for the context of remote or hybrid teaching. The methodological process consisted of the following activities: i) research, ii) selection, iii) curation, iv) adaptation and v) online catalog. As a result, the work presents the formulation of two initial criteria, used in a first pilot example and a questionnaire with new possibilities. As a future objective, it is intended to provide an online portfolio, with the activities already adapted.
\end{abstract}

Resumo. A Computação Desplugada tem sido referência para o ensino de disciplinas da Educação em Computação. Entretanto o advento da pandemia da Covid-19 alterou esse cenário. Assim, este artigo tem como objetivo apresentar um conjunto de critérios, que possam balizar a escolha e a adaptação de atividades desplugadas para o contexto de ensino remoto ou híbrido. O processo metodológico foi composto das seguintes atividades: i) pesquisa, ii) seleção, iii) curadoria, iv) adaptação e v) catálogo online. Como resultado, o trabalho apresenta a formulação de dois critérios iniciais, utilizados em um primeiro exemplo piloto e um questionário com novas possibilidades. Como objetivo futuro, pretende-se disponibilizar um portfólio online, com as atividades já adaptadas.

\section{Introdução}

Entre diversas abordagens que buscam estimular o ensino de Pensamento Computacional (PC), a utilização da Computação Desplugada (CD) tem sido descrita como um método de grande aceitação por parte dos docentes e discentes [Oliveira et al. 2018], entre outros fatores, por se tratar de uma estratégia lúdica, caracterizada por ensinar fundamentos de computação sem ou com pouquíssimo uso de computadores [Bell et al. 2011, de Souza and Lopes 2018, Oliveira et al. 2021].

No início de 2020, a Organização Mundial da Saúde (OMS) decretou o estado de pandemia devido ao vírus Sars-Cov-2 [WHO 2020]. Em decorrência disto, foram tomadas medidas de distanciamento e isolamento social como forma de prevenção. Este contexto de pandemia da Covid-19 motivou mudanças imediatas, com grande esforços de 
diversas áreas da sociedade, sendo uma delas a educacional, que abruptamente precisou se adequar em grande parte ao formato imediato de ensino remoto [Arruda 2020].

Diante disso, este trabalho propõe como objetivo principal uma nova abordagem, que é teórica e computacionalmente simples, baseada na adaptação de atividades de CD, que estimulem aprendizagem de PC, já propostas na literatura. As atividades serão adaptadas de forma que possam ser realizadas no contexto de ensino híbrido e remoto, considerando fatores como: a presença do professor em ambiente online e a dispersão geográfica de alunos, o que impossibilita a aplicação de atividades presenciais professor/alunos e alunos/alunos, que são a grande maioria delas. Nem todas as atividade desplugadas são passíveis de serem realizadas em contexto remoto. Algumas delas necessitam de dinâmicas em grupo realizadas no mesmo espaço físico.

Como resultado este trabalho apresenta um conjunto de possíveis critérios, que podem ser utilizados para classificar e posteriormente adaptar (ou não) atividades de CD. Além disso, temos por objetivo futuro fazer uso dos critérios, para reunir em um portfólio online, dezenas de atividades desplugadas, já adaptadas para o ensino híbrido e remoto. Não é de nosso conhecimento um portfólio assim e que seja gratuito, fato que justifica ainda mais a motivação deste trabalho.

\section{Referencial Teórico e Trabalhos Relacionados}

Nessa seção, apresentaremos um breve referencial teórico, bem como, uma série de trabalhos relacionados. [Bordini et al. 2016] destaca que a CD é uma estratégia de ensino de fundamentos da computação que visa apresentar esses conceitos de forma mais lúdica, sem a necessidade de se aprofundar em detalhes técnicos. Além disso ela pode ser aplicada para pessoas de todas as idades, desde o ensino fundamental até o ensino superior, abordando diferentes conhecimentos e experiências [Bordini et al. 2016, Oliveira et al. 2018, da Cruz et al. 2021]. Com isto, esta estratégia tem por objetivo fazer com que os entraves técnicos e equívocos sobre o que de fato é computação sejam devidamente esclarecidos [Lima et al. 2021]. Além de educar aos alunos sobre o que é Ciência da Computação, a CD proporciona aos alunos uma imersão nos conceitos do PC [Wing 2006]. Pesquisadores que são defensores do PC argumentam que existem formas de abordar problemas com base na Computação, que são valiosas para todos os alunos, independentemente se eles pretendem ou não estudar Ciência da Computação como uma especialização ou carreira [Wing 2014, Bell et al. 2009, Brackmann 2017, Li et al. 2020].

Para esta pesquisa alguns trabalhos recentes foram considerados relevantes no contexto de utilização da CD como um instrumento para o ensino do PC, principalmente por considerarem o período de ensino remoto emergencial no Brasil, com destaque para os seguintes de 2020 e 2021: [Medeiros et al. 2020], [Figueiredo et al. 2020], [Tavares et al. 2021] e [Sassi et al. 2021]. Os quatro trabalhos relatados possibilitaram a difusão da Ciência da Computação por meio da CD no contexto de educação remota causada pela pandemia. Em particular, acredita-se que o presente trabalho pode contribuir e agregar ainda mais valor à essas pesquisas recentes, visto que ele objetiva disponibilizar para a comunidade escolar de ensino básico, um catálogo com diversas opções de atividades desplugadas adaptadas para o ensino remoto, o que o difere dos trabalhos aqui relacionados. 


\section{Proposta}

Este trabalho faz uso de uma pesquisa qualitativa que por sua vez fará uso de uma abordagem de pesquisa com análise documental [Kripka et al. 2015] aliada ao processo de curadoria de conteúdo. A curadoria de conteúdo envolve o processo de buscar e de selecionar, entre a grande quantidade de informações disponíveis na web, um conjunto de conteúdos e apresentá-los de forma significativa e organizada em torno de um tema específico. O trabalho envolve pesquisar, selecionar, organizar e publicar informações. De acordo com [Bassani 2021], fazer curadoria de conteúdo não é apenas reunir links ou conteúdos; mas envolve colocá-los em um contexto de organização, anotação e apresentação.

A questão de pesquisa $(\mathrm{QP})$ que motiva e direciona a realização desta pesquisa é a seguinte: Como atividades já conhecidas de CD para o ensino de PC podem ser adaptadas considerando os aspectos do ensino híbrido e remoto?

Para alcançar o objetivo deste trabalho as seguintes etapas foram idealizadas: 1) pesquisar por atividades desplugadas; 2) aplicar filtros de seleção nas atividades encontradas; 3 ) compreender as particularidades e desafios do ensino remoto ou híbrido; 4) criar e validar critérios para classificação de atividades desplugadas; 5) classificar cada atividade selecionada como sendo: aplicável, não aplicável ou adaptável ao ensino remoto ou híbrido; 6) desenvolver adaptações para as atividades que forem passíveis de adaptação; 7) criar e compartilhar um catálogo gratuito de atividades desplugadas que possam ser aplicadas em contexto de aulas remotas ou híbridas.

\section{Resultados Preliminares}

Os documentos que foram considerados para fase de curadoria deste trabalho são atividades de CD propostas na literatura. A Tabela 1 lista um conjunto preliminar das fontes de atividades desplugadas já selecionadas, que encontram-se disponíveis na internet em sítios e materiais digitais, tais como livros e gibis. Durante o processo de busca das atividades, foram e serão selecionados materiais que apresentam referências na área de ensino de computação por meio de $\mathrm{CD}$, um outro fator importante também é que o acesso aos materiais seja fácil e gratuito.

Tabela 1. Composição preliminar de fontes das atividades desplugadas

\begin{tabular}{lll}
\hline Título & Formato & Referência \\
\hline Almanaque de Ciência da Computação & Gibi & {$[\overline{\text { Romero et al. 2019] }}$} \\
Computação e eu & Livro & {$[$ Santana et al. 2019] } \\
Computer Science Unplugged & Livro & {$[$ Unplugged 2019] } \\
CS Fundamentals Unplugged & Site & {$[\overline{\text { Code.org 2021 }}]$} \\
CS First Unplugged & Site & {$[\overline{\text { Google 2021] }}$} \\
Materiais Didáticos Desplugados & Fascículo & {$[\overline{\text { Marques et al. 2021 }}$} \\
Pensamento Computacional Brasil & Site & {$[$ Brackmann 2021 } \\
Unicamp Desplugada & Site & {$[$ Barichello 2021 } \\
\hline
\end{tabular}

A partir da compreensão das particularidades e desafios do ensino remoto, este trabalho propõe um conjunto de critérios, que possam ser norteadores para a classificação 
das atividades desplugadas disponibilizadas em três categorias: aplicável, não aplicável ou adaptável. Após a análise e classificação das atividades, para aquelas que forem classificadas como adaptáveis, serão elaboradas adaptações visando contemplar as características e as necessidades do ensino remoto ou híbrido. É possível observar na Figura 1 um exemplo de classificação e de escolha de adaptação aplicadas à três atividades usando dois critérios de classificação previamente.

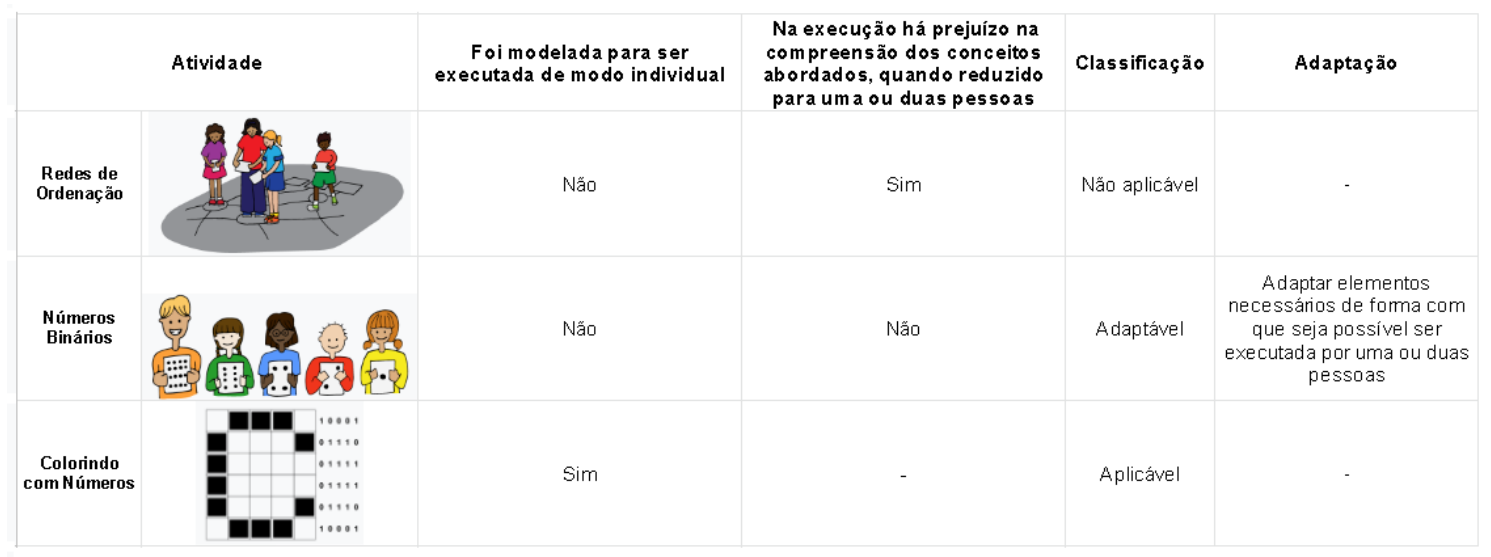

Figura 1. Exemplo de Classificação de Atividades

No exemplo demostrado, considera-se a questão do distanciamento físico, seja geográfico e/ou temporal entre alunos e professores, o que é uma característica do ensino remoto. Considerando o contexto nacional de exclusão digital, sugerimos o trabalho com as atividades desplugadas de forma assíncrona e de preferência individualizada. Assim sendo, alguns critérios já foram elaborados pelos autores, para a escolha e a adaptação das atividades selecionadas previamente: i) A atividade foi modelada para ser executada por um aluno, de modo individual. ii) Na execução da atividade há prejuízo na compreensão dos conceitos abordados caso seja reduzido o número de participantes para uma ou duas pessoas.

Para classificar a atividade, analisamos se corresponderia de forma positiva à primeira afirmação, caso a resposta seja sim, então a atividade será definida como aplicável. Respondendo não para os dois critérios, entende-se que para ela é possível realizar adequações e utilizá-la no ensino remoto ou híbrido desplugado proposto no catálogo. Quando a atividade apresenta característica contrária ao primeiro critério, porém, está de acordo com o segundo critério, é considerada como sendo não aplicável.

Ao analisar a atividade "Redes de Ordenação", conforme apresentado na Figura 1. o primeiro critério é a afirmação: "Esta atividade foi modelada para ser executada por um aluno, de modo individual". Neste caso, a atividade não corresponde ao critério analisado. O segundo critério é "Na execução desta atividade há prejuízo na compreensão dos conceitos abordados caso seja reduzido o número de participantes para uma ou duas pessoas", a atividade "Redes de Ordenação" concorda com este critério, por conta da necessidade de trabalho em grupo presencial, desta forma ela é classificada como não aplicável.

O segundo exemplo apresentado é a atividade "Números Binários". Esta atividade não condiz com a primeira afirmação, por ter sido modelada para ser aplicada a um grupo de alunos. No entanto, ela não concorda com o segundo critério podendo assim ser reali- 
zada por uma ou duas pessoas, sem prejuízos da compreensão dos conceitos abordados, sendo então classificada como adaptável. Já no terceiro exemplo, a atividade "Colorindo com Números", por sua vez, coincide com a primeira afirmação, pois foi formulada para ser realizada de forma individual. Em vista disso, automaticamente ela é classificada como aplicável.

Como observado, até agora apenas dois critérios foram formulados, entretanto para o prosseguimento do trabalho, seis novos critérios são propostos, em formato de questionário, acompanhados de duas questões abertas. Como representado na Tabela 2 , este questionário tem como objetivo medir a utilidade dos critérios para a classificação das atividades desplugadas nas modalidades de ensino remoto e híbrido. O instrumento é composto por escala Likert de cinco pontos [Likert 1932], sendo 1 nem um pouco útil e 5 extremamente útil. Na próxima etapa, pretende-se aplicar este questionário com diversos especialistas em CD, para aperfeiçoamento dos critérios e continuidade do trabalho de seleção, adaptação e construção do portfólio.

Tabela 2. Proposição de Questionário para referendar critérios

\begin{tabular}{lll}
\hline Id & Questões & Opções \\
\hline 1 & $\begin{array}{l}\text { A modelagem da atividade desplugada permite a execução por um único } \\
\text { aluno, sem comprometer o conteúdo trabalhado. }\end{array}$ & Escala Likert de cinco pontos \\
2 & $\begin{array}{l}\text { Quando realizada no ensino híbrido, a atividade desplugada permite a } \\
\text { execução de forma cooperativa entre os alunos. }\end{array}$ & Escala Likert de cinco pontos \\
3 & $\begin{array}{l}\text { A atividade desplugada exige produção de algum artefato por meio de } \\
\text { utilização de materiais de fácil acesso ao aluno. }\end{array}$ & Escala Likert de cinco pontos \\
4 & $\begin{array}{l}\text { A elaboração/construção do material pelo aluno é adequada a sua faixa } \\
\text { etária, de forma a possibilitar uma elaboração autônoma. }\end{array}$ & Escala Likert de cinco pontos \\
5 & $\begin{array}{l}\text { A atividade desplugada apresenta um grau de complexidade adequada, } \\
\text { possibilitando a realização autônoma do aluno. }\end{array}$ & Escala Likert de cinco pontos \\
6 & $\begin{array}{l}\text { A atividade desplugada apresenta um alto grau de complexidade, neces- } \\
\text { sitando em determinados momentos do acompanhamento do professor. }\end{array}$ & Escala Likert de cinco pontos \\
7 & $\begin{array}{l}\text { Dentre os critérios apresentados até agora, você tem alguma sugestão } \\
\text { de mudança e/ou melhoria? }\end{array}$ & Aberta \\
8 & $\begin{array}{l}\text { Caso você tenha em mente outros pontos importantes, registre aqui sua } \\
\text { contribuição sobre critérios para caracterizar uma atividade desplugada }\end{array}$ & Aberta \\
& no ensino remoto ou ensino híbrido. & \\
\hline
\end{tabular}

\section{Considerações Finais}

Durante o período de isolamento social causado pela pandemia da Covid 19, o ensino em geral, e claro, formato de atividades de CD precisou ser repensado para se adequar ao ensino remoto emergencial. No intuito de enfrentar esse desafio, esse estudo buscou apresentar critérios que possam balizar e futuramente oferecer a comunidade escolar um portfólio online inédito com diferentes atividades desplugadas, adaptadas ao contexto de ensino remoto ou híbrido. Os resultados preliminares já demostram a possibilidade do uso de CD no ensino híbrido. Como atividades futuras, temos por objetivo estabelecer, avaliar e disponibilizar o catalogo de atividades, bem como, promover ações de divulgação para promoção do ensino de PC no ensino híbrido. Desse modo, nosso estudo busca contribuir de maneira prática para diferentes atores ligados ao ensino de Computação. 


\section{Referências}

Arruda, E. P. (2020). Educação remota emergencial: elementos para políticas públicas na educação brasileira em tempos de covid-19. EmRede-Revista de Educação a Distância, $7(1): 257-275$.

Barichello, L. (2021). Unicamp desplugada. Disponível em: http://desplugada.ime.unicamp.br/. Acesso em: 25/04/2021.

Bassani, Patrícia B. Scherer; Magnus, E. B. (2021). Práticas de curadoria como atividades de aprendizagem na cultura digital. Sociedade Brasileira de Computação, Série Informática na Educação, v.1.

Bell, T., Alexander, J., Freeman, I., and Grimley, M. (2009). Computer science unplugged: School students doing real computing without computers. The New Zealand Journal of Applied Computing and Information Technology, 13(1):20-29.

Bell, T., Witten, I. H., Fellows, M., Adams, R., and McKenzie, J. (2011). Ensinando ciência da computação sem o uso do computador. Computer Science Unplugged ORG.

Bordini, A., Avila, C. M. O., Weisshahn, Y., da Cunha, M. M., da Costa Cavalheiro, S. A., Foss, L., Aguiar, M. S., and Reiser, R. H. S. (2016). Computaçao na educaçao básica no brasil: o estado da arte. Revista de Informática Teórica e Aplicada, 23(2):210-238.

Brackmann, C. P. (2017). Desenvolvimento do pensamento computacional através de atividades desplugadas na educação básica.

Brackmann, C. P. (2021). Pensamento computacional brasil. Disponível em: https://www.computacional.com.br/. Acesso em: 25/04/2021.

Code.org (2021). Cs fundamentals unplugged. Disponível em: https://code.org, Acesso em: $25 / 04 / 2021$.

da Cruz, M. E. K., Marques, S. G., and Oliveira, W. (2021). Desenvolvimento e avaliação de material didático desplugado para o ensino de computação na educação básica. Revista Brasileira de Informática na Educação, 29:160-187.

de Souza, J. S. and Lopes, A. S. B. (2018). Estimulando o pensamento computacional e o raciocínio lógico no ensino fundamental por meio da obi e computação desplugada. In Brazilian Symposium on Computers in Education (Simpósio Brasileiro de Informática na Educação-SBIE), volume 29, page 1893.

Figueiredo, L. A., Dias, A. B. A., Fagundes, L. A. G., de Paula Silva, V., Almeida, S. G. M., Magalhães, M. L. C., Rezende, P. M., Lima, M. O., Fortes, L. S., Resende, O. S., et al. (2020). Código x em casa: um relato de experiência sobre o ensino remoto de computação desplugada para meninas em situação de vulnerabilidade socioeconômica, em tempos de distanciamento social. In Anais do XXVI Workshop de Informática na Escola, pages 279-288. SBC.

Google (2021). Cs first unplugged. Disponível em: https://csfirst.withgoogle.com/ Acesso em: 25/04/2021.

Kripka, R., Scheller, M., and Bonotto, D. L. (2015). Pesquisa documental: considerações sobre conceitos e características na pesquisa qualitativa. CIAIQ2015, 2. 
Li, Y., Schoenfeld, A. H., diSessa, A. A., Graesser, A. C., Benson, L. C., English, L. D., and Duschl, R. A. (2020). Computational thinking is more about thinking than computing.

Likert, R. (1932). A technique for the measurement of attitudes. Archives of psychology.

Lima, A. C. d., Santos, Q. d. A., Junior, A. A. d. C., and Sanavria, C. Z. (2021). Mulheres na computação: Uma análise da participação de meninas em uma maratona gamificada e desplugada de pensamento computacional. Anais do Computer on the Beach, 12(0):384-391.

Marques, S. G., Schulz, F. H., and Cruz, M. d. (2021). Materiais didáticos desplugados. Disponível em: http://projetouid.weebly.com/materiais-didaticos-desplugados.html. Acesso em: 06/07/2021.

Medeiros, S. R., Martins, C. A., and Madeira, C. A. (2020). Contextualizando as atividades desplugadas para aumentar o engajamento das crianças. In Anais do XXXI Simpósio Brasileiro de Informática na Educação, pages 1543-1552. SBC.

Oliveira, W., Cambraia, A. C., and Hinterholz, L. T. (2021). Pensamento computacional por meio da computação desplugada: Desafios e possibilidades. In Anais do XXIX Workshop sobre Educação em Computação, pages 468-477. SBC.

Oliveira, W., Silva, F. C., Hinterholz, L. T., Isotani, S., and Bittencourt, I. I. (2018). Computaçao desplugada: Um mapeamento sistemático da literatura nacional. $R E-$ NOTE, 16(2):626-635.

Romero, M., Vallerand, V., and Nunes, M. (2019). Almanaque para popularização de ciência da computação. http://almanaquesdacomputacao.com.br/, 25(09):2019.

Santana, B. L., Araujo, L. G. J., and Bittencourt, R. A. (2019). Computação e Eu : Livro do Professor. Edição do Autor, Feira de Santana, 1 edition. https://sites.google.com/view/computacaofundamental/sextoano.

Sassi, S., Maciel, C., and Pereira, V. (2021). Computação (des)plugada: um relato de experiência sobre o ensino remoto de números binários em tempos de distanciamento social. In Anais do XXIX Workshop sobre Educação em Computação, pages 21-30, Porto Alegre, RS, Brasil. SBC.

Tavares, T. E., Marques, S. G., and da Cruz, M. K. (2021). Plugando o desplugado para ensino de computação na escola durante a pandemia do sars-cov-2. In Anais do Simpósio Brasileiro de Educação em Computação, pages 263-271. SBC.

Unplugged, C. (2019). Computer science without a computer.

WHO (2020). World health organization. Disponível em: https://www.who.int/emergencies/diseases/novel-coronavirus-2019. Acesso em: 06/05/2021.

Wing, J. (2014). Computational thinking benefits society. 40th anniversary blog of social issues in computing.[gs search].

Wing, J. M. (2006). Computational thinking. Communications of the ACM, 49(3):33-35. 